NASA-TM-111971

Optical logarithmic transformation of speckle images with bacteriorhodopsin films

John D. Downie

a reprint from Optics Letters 



\title{
Optical logarithmic transformation of speckle images with bacteriorhodopsin films
}

\author{
John D. Downie \\ NASA Ames Research Center, M/S 269-3, Moffett Field, California 94035
}

Received July 25, 1994

\begin{abstract}
The application of logarithmic transformations to speckle images is sometimes desirable in converting the speckle noise distribution into an additive, constant-variance noise distribution. The optical transmission properties of some bacteriorhodopsin films are well suited to implement such a transformation optically in a parallel fashion. I present experimental results of the optical conversion of a speckle image into a transformed image with signalindependent noise statistics, using the real-time photochromic properties of bacteriorhodopsin. The original and transformed noise statistics are confirmed by histogram analysis.
\end{abstract}

Logarithmic image transformations may be useful any time the noise on an image can be described as multiplicative in nature. ${ }^{1}$ This nonlinear operation on the image separates the noise from the underlying object function by producing a new image with additive noise. Traditional linear filtering techniques can then be applied for purposes of image restoration, etc. One commonly occurring type of multiplicative image noise is speckle, found during imaging with spatially coherent light or radiation when the object of interest has a random surface roughness of the order of a wavelength and the imaging system cannot resolve the microscale of the object's roughness. ${ }^{2}$ In addition to allowing the easy application of linear filters for image processing, use of a logarithmic function has been recently shown to significantly improve object detection and recognition of speckle images through correlation with a matched filter after preprocessing the image with a logarithmic transformation to make the noise additive and signal independent. ${ }^{3}$ In this Letter I propose and present experimental results of the optical logarithmic transformation of speckle images by using the real-time photochromic properties of bacteriohodopsin (BR). Although digital implementation is relatively easy, there may be situations such as the input of speckle images into an optical correlator in which it would be advantageous to perform all processing operations optically. BR films can accomplish this transformation in real time and in parallel and are thus an attractive option.

The statistical properties of speckle noise have been thoroughly studied, and it is well known that the image intensity generally follows a negative exponential probability-density function (pdf). ${ }^{4}$ An image model that has been widely used to describe speckle images is the multiplicative model,

$$
r_{\mathrm{sp}}(x, y)=\kappa s(x, y) n_{\mathrm{sp}}(x, y),
$$

where $r_{\mathrm{sp}}(x, y)$ is the noisy speckle image intensity, $s(x, y)$ is the incoherent intensity image of the object, and $n_{\mathrm{sp}}(x, y)$ is the noise function. ${ }^{1,5}$ We note that Eq. (1) is strictly true only for spatially uniform areas of the object. ${ }^{5}$ Assuming the model of Eq. (1) and negative exponential statistics for $n_{\mathrm{sp}}(x, y)$, it is easy to show that both the mean and the standard deviation of $r_{\mathrm{sp}}(x, y)$ are proportional to $s(x, y)$, which clearly makes the noise signal-dependent in nature. Because the logarithm of a product is mathematically equal to the sum of the individual logs, it is obvious that a logarithmic transformation of an image described by Eq. (1) will change the noise from multiplicative to additive. However, for speckle noise it also makes the new noise signal independent in nature. ${ }^{6}$ This considerably simplifies the image processing for restoration purposes and also produces an image better suited for correlation with a matched filter for pattern detection.

For digital image processing applications, the logarithmic operation would be performed digitally. However, for some optical processing applications, such as correlation, it may be more efficient to also preprocess the images in this logarithmic fashion optically. We then require an optical material that exhibits a logarithmic transfer function. BR is an organically derived material that demonstrates many desirable properties for use as a real-time optically addressed spatial light modulator for optical processing applications. ${ }^{7,8}$ It is a photochromic protein found in the photosynthetic system of a salt-marsh bacterium called Halobacterium salinarium. The BR molecule is contained in a membrane commonly called the purple membrane. The absorption of light initiates a photocycle in the BR molecule, which makes it a useful material as an optically addressed spatial light modulator. In the dark, the molecule is initially in the bR state, with an absorption spectrum peaked at $\sim 570 \mathrm{~nm}$. On absorption of a photon in this band, the molecule quickly passes through several intermediate states by thermal relaxation until it reaches the $M$ state, with its absorption spectrum shifted $\sim 160 \mathrm{~nm}$ towards the blue. Thus images may be written in a BR film based on the conversion of molecules from the bR state to the $\mathrm{M}$ state. In its native form, at nominal $\mathrm{pH}$, relative humidity, 


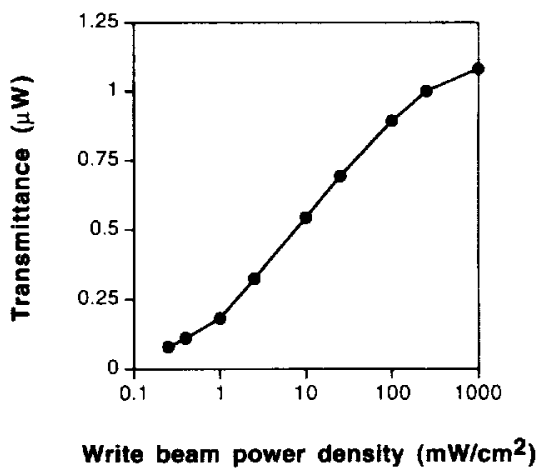

Fig. 1. Transmittance of read beam as a function of write beam power density for wild-type BR film. Both beams have wavelengths of $514.5 \mathrm{~nm}$.

and ambient temperature, the lifetime of the $\mathrm{M}$ state is $-10 \mathrm{~ms}$. However, the image lifetime can be increased by orders of magnitude by the use chemical ${ }^{8}$ and mutagenic ${ }^{\gamma}$ methods.

The film that we used in this experiment was prepared from the wild-type form of BR by Bend Research, Inc. The purple membrane material was extracted from the bacteria, suspended in a polymer matrix, and deposited upon an optical flat. The film was dried under controlled humidity conditions to lower the water content. The M-state lifetime was lengthened to $\sim 10 \mathrm{~s}$ by means of chemical additives, producing a solution $\mathrm{pH}$ of approximately 9.0. Finally, the film was sealed between the substrate and another glass plate. The film thickness was approximately $100 \mu \mathrm{m}$, and the optical density of the film in the bR state at the peak wavelength was 2.4 .

The light transmission characteristics of the film were measured experimentally by a variable-strength write beam and weak read beam whose intensity was small enough so as not to affect the transmission measurement. Both write and read beams were plane waves from an argon-ion laser at $514.5 \mathrm{~nm}$. The film was first exposed to the write beam, which caused part of the bR state population to traverse the photocycle and switch predominantly to the $M$ state. In a steady-state condition, the read beam was passed through the exposed area of the film and the transmitted intensity was measured with a photodetector. The data describing the relationship between transmitted intensity of the read beam, $I_{\text {read }}$, and the incident power density of the write beam, $E_{\text {write }}$, are given in Fig. 1. It is clear that the function is logarithmic over the central part of the curve, i.e.,

$$
I_{\text {trans }, \text { read }}=k \log \left(E_{\text {write }}\right)+c,
$$

for some constants $k$ and $c$. Furthermore, the dynamic range of the write power over which the transmittance of the BR film is logarithmic is large ( $>2$ orders of magnitude), which is required for the transformation of images with multiplicative noise such as speckle. Other films made from the wild-type form of $\mathrm{BR}$ also displayed behavior very similar to that shown in Fig. 1.

I then performed an experiment to optically implement a logarithmic image transformation with the
BR film. The optical setup is shown in Fig. 2. The argon laser beam is collimated and then split into two beams, each controlled with an electronic shutter. With shutter A open and shutter B closed, a transparency in contact with a diffuser is illuminated and an imaging system forms the speckle image of the transparency on the BR film. The power level of the exposing beam is set to ensure that we are operating in the central logarithmic region of the transmission curve of Fig. 1. Immediately after the speckle image is recorded on the film, shutter A is closed, shutter $B$ is opened, and the beam transmitted through the BR film is imaged into a CCD camera. The read beam power level is weak to ensure that it does not affect the image written in the BR film and also so that it is within the linear operating range of the CCD camera.

The performance of the BR film was evaluated with a two-tone object encoded on the transparency. The right half of the object is clear, and the left half is a gray level. The speckle image of this object as obtained at the $\mathrm{BR}$ film plane is shown in Fig. 3(a). The image obtained by the CCD camera on transmission of the read beam through the $\mathrm{BR}$ film after recording is shown in Fig. 3(b). I analyzed both images in Fig. 3 and determined the first- and second-order statistics; these results are presented in Table 1. For the speckle image, the equality of the mean and the standard deviation in each half is consistent with the model of a negative exponential pdf. On the other hand, the image transmitted by the BR

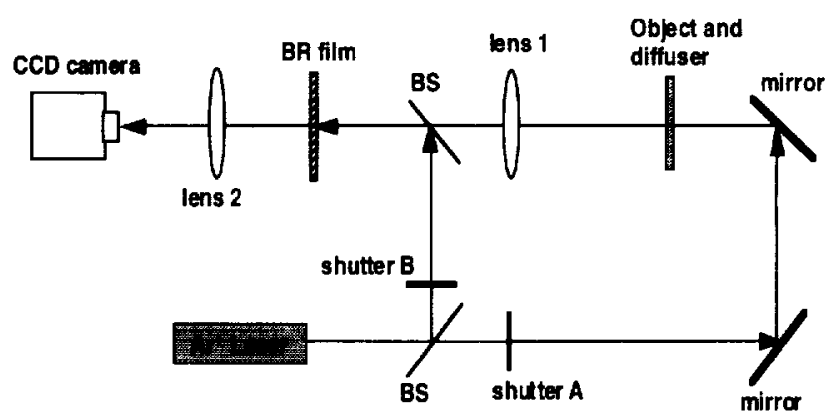

Fig. 2. Optical setup for logarithmic transformation of speckle image with BR film. Shutter A is opened during write process, and shutter $B$ is opened during read process. BS's, beam splitters.

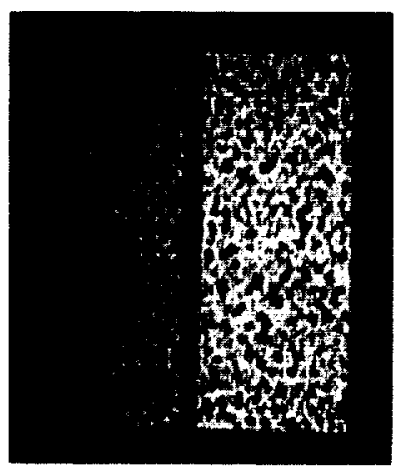

(a)

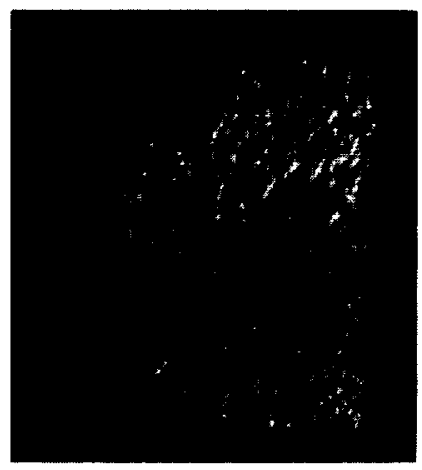

(b)
Fig. 3. (a) Original speckle image of a two-tone object, (b) logarithmically transformed image of the object obtained through BR film. 
Table 1. Image Statistics (Absolute Numbers Are Unrelated between Two Images)

\begin{tabular}{lcccr}
\hline & \multicolumn{2}{c}{ Speckle Image } & \multicolumn{2}{c}{ Image Transmitted by BR } \\
\cline { 2 - 3 } & Left Half & Right Half & & Left Half \\
\hline Mean, $\mu$ & 31.9 & 98.5 & 90.0 & Right Half \\
Standard Deviation, $\omega$ & 29.0 & 96.8 & 37.4 & 122.0 \\
\hline
\end{tabular}

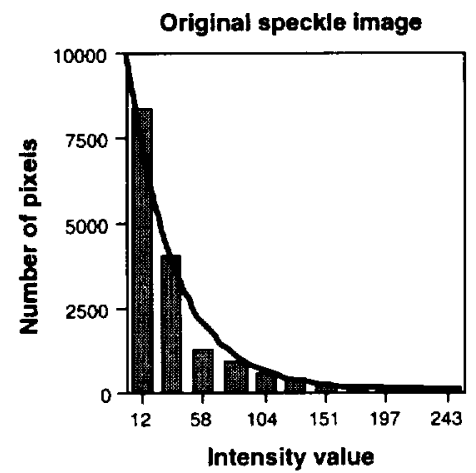

(a)

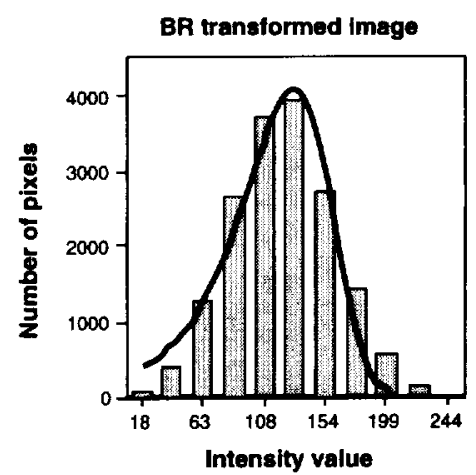

(b)

Fig. 4. (a) Histogram of the right half of a speckle image. (b) Histogram of the right half of the image transmitted by $\mathrm{BR}$ film. The solid black curves represent the predicted pdf's.

film shows remarkably different statistics; the means of the two halves are different, but the standard deviations are almost exactly equal. Thus the noise of the transmitted image is indeed signal independent. It can also be easily verified that the ratio of means of the two halves of the BR-transmitted image is consistent with that expected from the logarithmic transformation of the speckle image.

Histograms of the speckle image and the BRtransformed image are presented in Fig. 4 . The speckle image histogram closely resembles the expected negative exponential pdf of our speckle model. The transformed image histogram clearly shows that the new noise distribution is additive in nature. For a logarithmic transformation as given in Eq. (2), the expected pdf of the transformed image noise $n$ is of the form

$$
p_{N}(n)=\alpha 10^{n / k} \exp \left(-\gamma 10^{n / k}\right)
$$

for some constants $k, \alpha$ and $\gamma$. The pdf predicted by Eq. (3) is slightly more asymmetric, with a longer tail to the left-hand side than the histogram data in Fig. 4(b), but the experimental results are nevertheless quite similar to those predicted. The relatively minor differences can probably be explained by experimental noise and measurement error that tend to make the real distribution more Gaussian than the purely theoretical model.

I have experimentally demonstrated the optical logarithmic transformation of an image by using bacteriorhodopsin film. In particular, the multiplicative, signal-dependent noise of a speckle image has been transformed into additive, signal-independent noise. Given that BR films have high spatial resolution, require no external processing, and may be cycled through the write/read/erase process millions of times without degradation, such films have the potential to function as a rapid, parallel optical logarithmic transformer for images with multiplicative noise such as may be required in the operation of an optical correlator or in general preparation for subsequent optical image processing algorithms.

This work was supported by the NASA Office of Advanced Concepts and Technology under RTOP 23302-05-06. The author thanks Dan Smithey of Bend Research, Inc., for helpful discussions.

\section{References}

1. H. Kato and J. W. Goodman, Appl. Opt. 14,1813 (1975).

2. J. W. Goodman, in Laser Speckle and Related Phenomena, J. C. Dainty, ed., Vol. 9 of Topics in Applied Physics (Springer-Verlag, New York, 1975), p. 10.

3. J. D. Downie and J. F. Walkup, J. Opt. Soc. Am. A 11, 1599 (1994).

4. J. W. Goodman, Statistical Optics (Wiley, New York, 1985), Chap. 7, p. 350.

5. M. Tur, K. C. Chin, and J. W. Goodman, Appl. Opt. 21, 1157 (1982)

6. H. H. Arsenault and M. Denis, Opt. Lett. 6, 210 (1981).

7. C. Bräuchle, N. Hampp, and D. Oesterhelt, Adv. Mater. 3, 420 (1991).

8. R. R. Birge, Annu. Rev. Phys. Chem. 41, 683 (1990). 
